\title{
A Case of Reynolds Syndrome: Scleroderma and Primary Biliary Cirrhosis Overlap Syndrome
}

\author{
Marco Simoes ${ }^{\mathrm{a}, \mathrm{c}}$, Patricia Alves ${ }^{\mathrm{a}}$, Helder Esperto ${ }^{\mathrm{a}}$, Benilde Barbosa ${ }^{\mathrm{a}}$, Lelita Santos ${ }^{\mathrm{a}}$, \\ Jose Manuel Nascimento Costa,
}

\begin{abstract}
Primary biliary cirrhosis (PBC) is a chronic liver condition characterized by bile duct destruction, probably caused by a yet unknown immunological mechanism. Its association with several other autoimmune diseases has been described. The association of the limited cutaneous form of systemic scleroderma (lcSSc) and PBC is known as Reynolds syndrome. The authors describe a case of a 76 year old male patient with Raynaud's phenomenon complaints in 1999. The physical examination revealed sclerodactyly, cutaneous calcifications, digital ulcers and facial telangiectasias. Chest radiography showed signs of lung fibrosis. Dilated capillary loop were found in nailfold capillaroscopy. The autoimmunity pattern was compatible with limited systemic sclerosis (antinuclear and anticentromere antibodies were positive). In 2005 the autoimmunity pattern changed, with positive antimitochondrial antibodies. The patient presented with fatigue, pruritus and dyspnea on exertion complaints. Liver function tests showed elevation of alkaline phosphatase and gamma-glutamyl transpeptidase. Although the patient refused liver biopsy, PBC diagnosis was established. In 2008 the patient was admitted to the hospital with anorexia, dyspepsia, ascites, abdominal pain and weight loss. Endoscopy showed esophageal varices and abdominal computed tomography revealed cirrhotic liver. The patient died in 2009 with acute myocardial infarction.
\end{abstract}

Keywords: Limited systemic cutaneous sclerosis; CREST syndrome; Primary biliary cirrhosis; Overlap syndrome; Reynolds syndrome; Autoimmunity; Nailfold capillaroscopy; Raynaud's

Manuscript accepted for publication August 10, 2011

anternal Medicine Department, Hospitais da Universidade de Coimbra, Portugal

${ }^{\mathrm{b}}$ Faculdade de Medicina da Universidade de Coimbra, Portugal

${ }^{\mathrm{C}}$ Corresponding author: Marco Simoes, Urb. Quinta das Lagrimas, Fase

2, Lote 2, 4 A, 3040-387 Coimbra, Portugal.

Email: marcosimoes@gmail.com

doi:10.4021/jmc276w phenomenon

\section{Introduction}

Primary biliary cirrhosis (PBC) is a chronic and progressive disease probably caused by a yet unknown immunological mechanism. Its hallmark is the destruction of septal and interlobular ducts and portal inflammation with scarring, causing extensive fibrosis and liver failure [1-3].

Systemic sclerosis (SSc) is a connective tissue disease characterized by inflammatory, fibrotic and degenerative changes. It may be classified as diffuse cutaneous SSc or limited cutaneous SSc (lcSSc) on the basis of the extent and distribution of skin involvement. The systemic manifestations of the disease include circulation abnormalities, such as Raynaud's phenomenon, and multiple organ involvement with fibrotic and vascular complications [3-5].

The association between PBC and other autoimmune conditions, such as Sjögren's syndrome, rheumatoid arthritis, Hashimoto's thyroiditis or systemic sclerosis occurs in $84 \%$ of cases [1, 2, 6-10]. The overlap of lcSSc and PBC is known as Reynolds syndrome, after the lead author of an article published in 1971 [11]. The authors report a patient with lcSSc and PBC.

\section{Case Report}

In 1999 a 76-year-old man presented with Raynaud's phenomenon complaints and mild arthralgias in the previous month. His past medical history revealed only benign prostatic hyperplasia and arterial hypertension. Physical examination showed mild skin thickening of forearms and moderate skin thickening of hands (according to modified Rodnan skin score), sclerodactyly, cutaneous calcifications in the forearms, pitting digital ulcers in the 2nd and 3rd fingers of the right hand and facial telangiectasias. Thoracic and abdominal examination was normal.

The routine laboratory workup (hemogram, ionogram, liver function tests, c-reactive protein and erythrocyte sedi- 


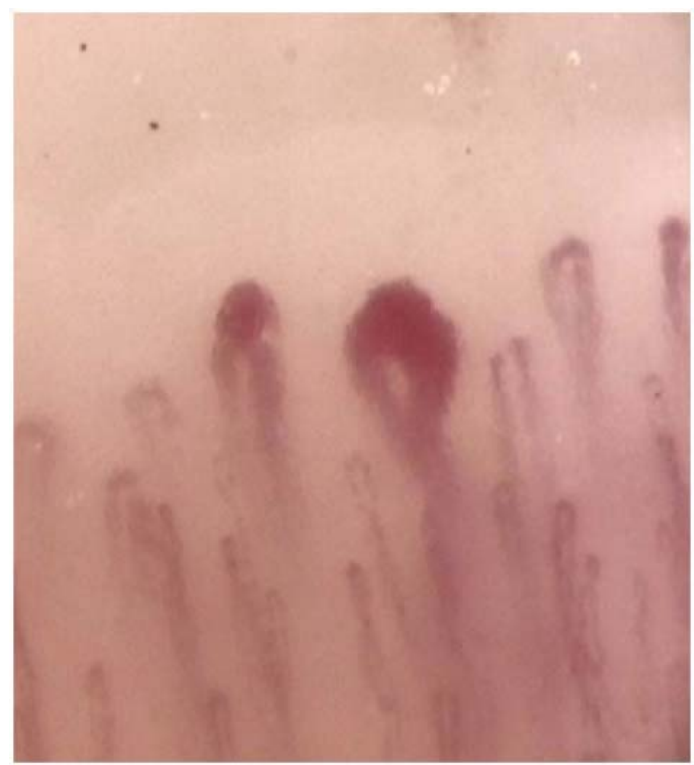

Figure 1. Nailfold capillaroscopy showing dilated capillaries and one megacapillary, 200x magnification, 1999.

mentation rate) was normal. Immunoelectrophoresis was suggestive of monoclonal gammopathy IgM kappa. The proteinogram revealed gamma monoclonal peak. Bone marrow examination detected changes compatible with monoclonal gammopathy of undetermined significance (MGUS). Antinuclear and anticentromere antibodies were positive (with maximum score in a qualitative scale, ++++ ). Anti-SSA(Ro) antibody was also positive. All other autoimmune tests were negative. Chest radiography had discrete signs of lung fibrosis. Nailfold capillaroscopy showed senile pattern in the left hand, while in the right hand it showed dilated capillaries, few giant capillaries, severe stasis and pericapillar edema, without avascular areas (Fig. 1). Limited cutaneous systemic sclerosis diagnosis was established. Treatment with sustained-release nifedipin $30 \mathrm{mg}$ id, aspirin $100 \mathrm{mg}$ id and pentoxifylline $400 \mathrm{mg}$ bid was begun, with clinical improve- ment.

The patient was regularly observed, with no changes in the autoimmunity pattern until 2005, when in addition to the antinuclear and anticentromere, antimitochondrial antibodies (AMA) were also strongly positive $(++++)$. Liver function tests, particularly alkaline phosphatase, were normal, while erythrocyte sedimentation rate was $45 \mathrm{~mm} /$ hour. In 2006 he presented with fatigue, pruritus and dyspnea on exertion. Liver function tests showed elevation of alkaline phosphatase (143 U/L, normal range 30 - $120 \mathrm{U} / \mathrm{L}$ ) and gamma-glutamyl transpeptidase (81 U/L, normal range $<55$ $\mathrm{U} / \mathrm{L})$. Although the patient refused liver biopsy, two PBC criteria were met and the diagnosis was established. Treatment with ursodeoxycholic acid $250 \mathrm{mg}$ tid and cholestyramine 4 gr bid was instituted. Mild increase in liver function tests was observed over time (Table 1).

In 2008 the patient was admitted to the hospital with 4 months history of anorexia, dyspepsia, abdominal pain and significant weight loss (27\% of body weight lost in that period). On physical examination the patient presented abdominal distention and ascites. Laboratory workup revealed low serum albumin (3.3 g/dL, normal range 3.5 - 5.2 g/dL), elevated alkaline phosphatase (215 U/L), gamma-glutamyl transpeptidase (83 U/L) and aspartate aminotransferase (45 $\mathrm{U} / \mathrm{L}$, normal range $<35 \mathrm{U} / \mathrm{L}$ ). Endoscopy showed grade II esophageal varices in the distal third of the esophagus and congestive and hypertrophic gastric mucosa. Abdominal computed tomography was performed, presenting small nodular liver, with difuse steatosis, and splenomegaly. Furosemide $40 \mathrm{mg}$ bid, spironolactone $50 \mathrm{mg}$ id and propranolol $20 \mathrm{mg}$ bid were added to the previous therapeutic regimen with clinical improvement. Simvastatin $20 \mathrm{mg}$ id was also prescribed for the treatment of recently diagnosed hypercholesterolemia.

The patient was admitted to the emergency room in 2009 with chest pain. Acute myocardial infarction was diagnosed and standard medical therapy with aspirin, clopidogrel and low molecular weight heparin was given, but the patient died

Table 1. Liver Function Tests

\begin{tabular}{llllllllllll}
\hline \multicolumn{1}{c}{ Item } & Normal & $\mathbf{2 0 0 0}$ & $\mathbf{2 0 0 1}$ & $\mathbf{2 0 0 2}$ & $\mathbf{2 0 0 3}$ & $\mathbf{2 0 0 4}$ & $\mathbf{2 0 0 5}$ & $\mathbf{2 0 0 6}$ & $\mathbf{2 0 0 7}$ & $\mathbf{2 0 0 8}$ & $\mathbf{2 0 0 9}$ \\
\hline AST (U/L) & $<35$ & 25 & 31 & 30 & 31 & 21 & 30 & 37 & 67 & 45 & 45 \\
ALT (U/L) & $<45$ & 11 & 15 & 15 & 16 & 12 & 17 & 24 & 49 & 22 & 24 \\
Alkal Ph (U/L) & $30-120$ & 73 & 101 & 93 & 116 & 101 & 104 & 143 & 242 & 215 & 188 \\
GGT (U/L) & $<55$ & 22 & 31 & 30 & 35 & 27 & 52 & 81 & 145 & 83 & 63 \\
TBrb (mg/dL) & $0.3-1.2$ & 0.5 & 0.5 & 0.5 & 0.4 & 0.5 & 0.6 & 0.5 & 0.7 & 0.8 & 1.0 \\
\hline
\end{tabular}

AST - Aspartate aminotransferase; ALT - Alanine aminotransferase; Alkal Ph - Alkaline phosphatase; GGT - Gammaglutamyl transpeptidase; TBrb - Total bilirubin. 
before angioplasty could be performed.

\section{Discussion}

The overlap of different autoimmune conditions is widely known. The association of lcSSc and PBC was first described in 1970 by Murray-Lion et al [12] and in 1971 by Reynolds et al [11]. The prevalence of SSc in patients with PBC is 3-8\%. Conversely, the estimated prevalence of PBC among patients with SSc is 2.5-3\% [13]. Although the subjacent immune mechanisms are not yet completely known, Mayo et al reported that antigen-stimulated T-cells play an important role and described bigger prevalence of clonal populations of CD8+ TCRBV3 in Reynolds syndrome than in both conditions alone [14].

PBC is more frequent in women (8:1 female-to-male ratio) and patients present usually between the 5th and 7th decades, with an estimated incidence of 0.7 - 49 cases per million-population per year [2]. Systemic sclerosis also affects mostly women (5:1 female-to-male ratio), generally between the 3rd and 5th decades and with annual incidence of 14.1 cases per million [4]. The authors present a case of a male patient who first was diagnosed with lcSSc at the age of 76 years and with PBC seven years later. The onset of any of these diseases separately is uncommon either in the male gender or in older patients.

PBC is probably the result of the interaction between genetic background and extrinsic factors. Most patients are asymptomatic (48-60\%) [1, 6] and the diagnosis is suspected in routine blood tests, due to abnormal alkaline phosphatase values. Fatigue and pruritus are the most common symptoms $[1,2]$. According to the American Association for the Study of Liver Diseases (AASLD) guidelines on PBC, the diagnosis can be established when two out of three criteria: 1) biochemical evidence of cholestasis based mainly on alkaline phosphatase elevation; 2) presence of AMA; 3) histologic evidence of nonsuppurative destructive cholangitis and destruction of interlobular bile ducts [15]. Although liver biopsy allows diagnostic confirmation and disease staging, it is not mandatory for diagnosis once the other two criteria are met. In our case the patient had alkaline phosphatase elevation and positive AMA, therefore the diagnosis was established. Liver biopsy was considered, but the patient refused the procedure. Treatment with ursodeoxycholic acid was iniciated but liver function tests gradually worsened and finally in 2008 he was admitted due to liver failure. Liver transplant is the only treatment that truly improves PBC's natural history [1].

The diagnosis of lcSSc was based on clinical findings of severe Raynaud's phenomenon, mild calcinosis, sclerodactily and telangiectasia. Only esophageal dysmotility was lacking for the diagnosis of CREST syndrome, although the patient had dyspeptic complaints. Autoimmune pattern was also compatible with lcSSc, antinuclear and anticentromere antibodies were strongly positive and anti-DNA topoisomerase I (Scl-70) antibody was negative. Nailfold capillaroscopy revealed also typical findings of scleroderma [16, 17]. Symptomatic treatment for Raynaud's phenomenon was given to our patient, with positive evolution. SSc can cause heart disease, namely pericardial effusion, myocardial fibrosis and arrhythmias [4]. Despite this patient had no previous history of cardiac disease, he died of an acute myocardial infarction. We cannot exclude that this patient had cardiac manifestations of SSc.

Reynolds syndrome prognosis depends of PBC's severity, since lcSSc is a relatively benign slow progressive form of SSc [18]. The regular evaluation of liver function tests is useful for monitoring disease progression. Advanced age, hyperbilirubinemia, low serum albumin and stage 4 (cirrhosis) liver biopsy indicate worse prognosis $[1,2]$. Nevertheless, survival of patients with PBC and CREST syndrome seems to be better than that of patients with PBC alone [19].

\section{Conflict of Interest}

None

\section{References}

1. Kaplan MM, Gershwin ME. Primary biliary cirrhosis. N Engl J Med. 2005;353(12):1261-1273.

2. Kumagi T, Heathcote EJ. Primary biliary cirrhosis. Orphanet J Rare Dis. 2008;3:1.

3. Sahin M, Sarýtas U, Ozkan N, Tunc S, Karahan N. A case of primary biliary cirrhosis, CREST and Sjögren's syndrome overlap presented with severe esophageal variceal bleeding. S.D.Ü. Týp Fak Derg. 2007;14(2):38-42

4. Vlachoyiannopoulos PG. Systemic sclerosis (scleroderma). Orphanet encyclopedia, November 2001.

5. Vilas AP, Veiga MZ, Abecasis P. Esclerose sistémica perspectivas actuais. Med Interna 2002;9(2):111-120.

6. Valle L, Valente P, Lopes AV, Vaz AL. Síndrome de Reynolds: associação entre cirrose biliar primária e síndrome de CREST. Rev Port Reumatol Patol Osteo Art. 2002;13(128):6-14.

7. Ito M, Kojima T, Miyata M, Saka M, Kokubun M, Ohira $\mathrm{H}$, Ishikawa H, et al. Primary biliary cirrhosis (PBC)CREST (calcinosis, Raynaud's phenomenon, esophageal dysfunction, sclerodactyly and telangiectasia) overlap syndrome complicated by Sjogren's syndrome and arthritis. Intern Med. 1995;34(5):451-454.

8. Shoji I, Takagi T, Kasukawa R. Anti-centromere antibody and CREST syndrome in patients with primary biliary cirrhosis. Intern Med. 1992;31(12):1348-1355.

9. Marasini B, Gagetta M, Rossi V, Ferrari P. Rheumatic 
disorders and primary biliary cirrhosis: an appraisal of 170 Italian patients. Ann Rheum Dis. 2001;60(11):10461049.

10. Clarke AK, Galbraith RM, Hamilton EB, Williams R. Rheumatic disorders in primary biliary cirrhosis. Ann Rheum Dis. 1978;37(1):42-47.

11. Reynolds TB, Denison EK, Frankl HD, Lieberman FL, Peters RL. Primary biliary cirrhosis with scleroderma, Raynaud's phenomenon and telangiectasia. New syndrome. Am J Med. 1971;50(3):302-312.

12. Murray-Lyon IM, Thompson RP, Ansell ID, Williams R. Scleroderma and primary biliary cirrhosis. Br Med J. 1970;3(5717):258-259.

13. Sakamoto N, Ishimatsu Y, Kakugawa T, Hara A, Hara S, Amenomori M, Fujita H, et al. Sarcoidosis in a patient with systemic sclerosis and primary biliary cirrhosis. Intern Med. 2010;49(15):1609-1611.

14. Mayo MJ, Jenkins RN, Combes B, Lipsky PE. Associa- tion of clonally expanded $\mathrm{T}$ cells with the syndrome of primary biliary cirrhosis and limited scleroderma. Hepatology. 1999;29(6):1635-1642.

15. Lindor KD, Gershwin ME, Poupon R, Kaplan M, Bergasa NV, Heathcote EJ. Primary biliary cirrhosis. Hepatology. 2009;50(1):291-308.

16. Santos L, Fonseca I, Saldanha MH. Usefulness of nailfold capillary microscopy in the diagnosis of Raynaud's phenomenon. Med Interna 2010;17(3):141-150.

17. Cortes S, Cutolo M. Capillarosecopic patterns in rheumatic diseases. Acta Reumatol Port. 2007;32(1):29-36.

18. Krieg T, Takehara K. Skin disease: a cardinal feature of systemic sclerosis. Rheumatology (Oxford). 2009;48 Suppl 3:iii14-18.

19. Tojo J, Ohira H, Suzuki T, Takeda I, Shoji I, Kojima $\mathrm{T}$, Takagi $\mathrm{T}$, et al. Clinicolaboratory characteristics of patients with primary biliary cirrhosis associated with CREST symptoms. Hepatol Res. 2002;22(3):187-195. 